\title{
Receptor interacting protein kinase 3 promotes cisplatin-induced necroptosis in apoptosis-resistant HepG2/DDP cells
}

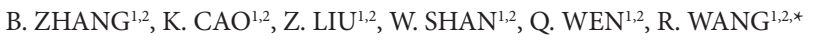 \\ ${ }^{1}$ Institute of Digestive Diseases, Xuzhou Medical University, Xuzhou, Jiangsu, China; ${ }^{2}$ Department of General Surgery, Affiliated Hospital of \\ Xuzhou Medical University, Xuzhou, Jiangsu, China \\ ${ }^{*}$ Correspondence: drwangrenhao@163.com
}

Received July 10, 2018 / Accepted February 14, 2019

\begin{abstract}
Hepatocellular carcinoma is the most common primary malignancy of the liver. The chemotherapeutic drug cisplatin is widely used for advanced liver cancer. However, the development of cisplatin resistance in cancer cells, which is related to the decreased cellular susceptibility to apoptosis, results in a major limitation of cisplatin-based chemotherapy. Recently, triggering necroptosis has been proposed to be a novel therapeutic strategy to eradicate apoptosis-resistant cancer cells. In this study, we provided evidence that cisplatin could induce cell death in HepG2 cells, but not in the apoptosis-resistant HepG2/DDP cells. Ectopic expression of RIP3 promoted cisplatin-induced HepG2/DDP cells death, HMGB1 and LDH release. Moreover, we demonstrated that this type of cell death was necroptosis and depended on RIP1-RIP3-MLKL signaling pathway because inhibition of MLKL activity by necrosulfonamide (NSA) or knockdown of RIP1 significantly attenuated cisplatin-induced cell death in HepG2/DDP-RIP3 cells. Finally, we found that ectopic expression of RIP3 sensitized HepG2/DDP cancer cells to cisplatin treatment in vivo. The findings offer new insights into the molecular mechanisms underlying cisplatin-induced necroptosis in liver cancer cells and suggest that combination of cisplatin with other drugs which can restore RIP3 expression in cancer cells maybe a better choice for therapy of apoptosis-resistant cancer.
\end{abstract}

Key words: hepatocellular carcinoma, cisplatin, necroptosis, RIP3

Two major forms of cell death have been fully defined: apoptosis and necroptosis [1]. They are characterized by morphological and biochemical differences. Apoptosis is involved in various life processes including normal embryonic development, innate immune response and tissue homeostasis. Previous research indicated that abnormal apoptosis contributed to neurodegenerative diseases, developmental malformation, autoimmune disorders or many types of cancer [2]. Apoptosis can be triggered by two main apoptotic pathways: the extrinsic pathway and the intrinsic pathway. The extrinsic pathway is initiated through activation of death receptor like tumor-necroptosis factor (TNF)-receptor 1 or Fas/CD95. Alternatively, intrinsic stresses such as direct DNA damage, hypoxia and growth factor deprivation, can activate the intrinsic apoptotic pathway. Both signaling pathways result in the activation of a cascade system of proteases, named caspases, which are the executioners of apoptotic program [3]. Apoptotic cells were characterized by nuclear condensation and DNA fragmentation, degradation of cellular proteins and organelles and apoptotic bodies formation [4]. Cellular necroptosis has long been described as an unexpected and uncontrolled type of cell death, which was caused by many chemical or physical events including radiation, heat, toxin, hypoxia, and so on. To date, necroptosis has been identified as a regulated cell death, which can be triggered by a variety of initiators, including death ligands (TNF- $a$, TRAIL and Fas), Toll like receptors ligands, interferons and HSV virus infection, when caspase activation was inhibited in a number of cell types [5-7]. Necroptosis was characterized by cytoplasmic swelling, rupture of organelle membranes and release of cellular contents into the extracellular environment $[8,9]$. The TNF family cytokines induced necroptosis required the activity of receptor-interacting protein kinase 1 (RIP1) and kinase RIP3. RIP3 has been identified as the central necroptosis mediator, which switched TNFa-induced apoptosis to necroptosis $[10,11]$. Activation of TNF $\alpha$ receptor induces ubiquitination of RIP1 and recruits TRADD, cIAPs, TRAF2 to form the pro-survival complex at membrane. When RIP1 is deubiquitinated by CYLD, RIP1 is translocated from membrane to cytoplasm and binds with FADD, caspase-8 and RIP3 to activate apoptosis. Inactivation of caspase-8 leads to RIP3-dependent necroptosis [12]. 
Mixed lineage kinase domain-like protein (MLKL) has been identified as a downstream substrate of RIP3. RIP3-mediated phosphorylation of MLKL promoted MLKL oligomerization, which disrupted membrane integrity during necroptosis. RIP1, RIP3 and MLKL formed a large multiprotein complex called necrosome which was required for execution of TNFa-induced necroptosis [13].

Irradiation and chemotherapeutic drugs for cancer therapy resulted in DNA damage-triggered signaling and induction of apoptosis, which was executed by intrinsic apoptotic pathway [14]. Recently, researches validated that necroptosis can be induced by classic necroptosis triggers or chemotherapeutic agents in a plethora of cancer cell lines including hepatocarcinoma, glioblastoma, leukemia, bladder carcinoma, and so on $[15,16]$. The chemotherapy drug cisplatin can induce caspase-dependent apoptosis in cancer cells and has been used for a number of solid tumor treatments, including human hepatocellular carcinoma. Moreover, other cell death pathway was also activated by cisplatin and other chemotherapeutic drugs [17]. For example, shikonin treatment induced cells death in C6 and U87 glioma cells and 12-PE, RPMI-8226 and U266 myeloma cells through necroptosis, and this death could be blocked by RIP1 inhibitor necrostatin-1 (Nec-1) [18]. Previous research showed that cisplatin caused gastric cancer cells necroptosis under inhibition of apoptosis [19]. Esophageal cancer cells were susceptible to cisplatin-initiated necroptosis when the apoptotic pathway was absent or blocked [20].

Now most cancer therapeutic agents aimed at an induction of apoptosis, however cancer cells usually evolved and got the ability to evade apoptosis [21]. Multiple drug resistance to cisplatin-based therapy was found in human hepatocellular carcinoma cells [22]. So it is desired to develop some new therapeutic strategies focusing on triggering other type of tumor-selective cell death. Cisplatin has been demonstrated to induce both apoptosis and necroptosis in some cancer cells, but whether cisplatin could induce necroptosis in hepatocellular carcinoma cells is largely unknown.

In the current study, two cell lines, HepG2, a human liver cancer cell line and HepG2/DDP, its cisplatin-resistant counterpart were analyzed. HepG2/DDP cells are resistant to cisplatin-induced apoptosis because of anti-apoptotic genes upregulation. We also found that RIP3, the central molecule in necroptosis is also absent in HepG2/DDP cells. Ectopic expression of RIP3 results in cisplatin-induced HepG2/DDP cells necroptosis via autocrine production of TNF- $\alpha$. More importantly, we demonstrate that cisplatin-induced necroptosis in HepG2/DDP-RIP3 cells requires RIP1 and MLKL because inhibition of MLKL activity by NSA or knockdown of RIP1 significantly attenuates cells death and leads to cisplatin resistance. Moreover, we report that HepG2/DDP-RIP3 cells are more sensitive to cisplatin treatment in vivo. These data provide new insight into the molecular mechanisms underlying cisplatin-induced necroptosis in liver cancer cells chemotherapy.

\section{Materials and methods}

Reagents. Cisplatin was purchased from Selleck. CellTiterGlo Luminescent Cell Viability Assay kit was from Promega. Nec-1 (necrostatin-1) was purchased from Sigma. DMEM medium and fetal bovine serum (FBS) were purchased from Gibco. RIP1, RIP3 and MLKL antibody were from Abcam. GAPDH antibody was purchased from Sigma.

Cell line and culture. HepG2 and HepG2/DDP cells were obtained from Shanghai Institute of Cell Biology, Chinese Academy of Sciences (Shanghai, China). Cells were cultured in DMEM supplemented with $10 \%$ fetal bovine serum, penicillin $(100 \mathrm{U} / \mathrm{ml})$ and streptomycin $(100 \mathrm{mg} / \mathrm{ml}), 37^{\circ} \mathrm{C}$ and $5 \% \mathrm{CO}_{2}$ in a humid environment.

Real-time PCR. Total RNA was prepared using TRIzol (Invitrogen). Reverse transcription was performed using PrimeScript $^{\text {th }}$ RT Master Mix (Takara, RR036A) according to the manufacturer's instructions. SYBR Fast qPCR Mix (RR430A) was purchased from Takara Bio Inc. PCR was performed on CFX96 (Bio-Rad). All mRNA expression levels were normalized to GAPDH. Each sample was run at least in triplicate. Primers used for the amplification are: GAPDH-F: 5'-CTGGGCTACACTGAGCACC GAPDH-R: 5'-AAGTGGTCGTTGAGGGCAATG, Bad-F: 5'-CCCAGAGTTTGAGCCGAGTG，Bad-R: 5'-CCCATCCCTTCGTCGTCCT. Bim-F: 5'-TAAGTTCTGAGTGTGACCGAGA, Bim-R: 5'-GCTCTGTCTGTAGGGAGGTAGG. Bak-F: 5'-CATCAACCGACGCTATGACTC, Bak-R: 5'-GTCAGGCCATGCTGGTAGAC. Bcl-XL-F: 5'-GAGCTGGTGGTTGACTTTCTC, Bcl-XL-R: 5'-GAGCTGGTGGTTGACTTTCTC. Bcl-2-F: 5'-GGTGGGGTCATGTGTGTGG, Bcl-2-R: 5'-CGGTTCAGGTACTCAGTCATCC. Bcl-W-F: 5'-GCGGAGTTCACAGCTCTATAC, Bcl-W-R: 5'-AAAAGGCCCCTACAGTTACCA.

Cell viability assay. Cells (2000/well) were seeded into 96-well plate and cultured 24 hours. Cisplatin was added into experimental group to reach the final concentration. DMSO was added to the control group. After cisplatin treatment at indicated time point, cellular viability was assessed using CellTiter-Glo Luminescent Cell Viability Assay according to the manufacturer's manual.

Measurement of HMGB1 and LDH by ELISA. $2 \times 10^{4}$ HepG2/DDP-Vector or HepG2/DDP-RIP3 cells were seeded in 96-well plate. The cells were treated with DMSO or $10 \mu \mathrm{M}$ cisplatin for 48 hours. HMGB1 in the supernatants was measured with HMGB1 ELISA kit (Shino Test Corporation) according to the instructions. $\mathrm{LDH}$ in the supernatants were determined with a LDH ELISA Kit (Abcam) according to the manufacturers' protocols.

Western blot analysis. Cells were collected by centrifugation at $1200 \mathrm{rpm}$ for $5 \mathrm{~min}$ and suspended in lysis buffer (20 mM Tris-HCl, pH 7.4, 150 mM NaCl, 10\% glycerol, $1 \%$ Triton X-100, $1 \mathrm{mM} \mathrm{Na} \mathrm{VO}_{4}, 25 \mathrm{mM} \beta$-glycerol-phosphate, $0.1 \mathrm{Mm}$ PMSF, a complete protease inhibitor set) then lysed on ice for $20 \mathrm{~min}$. Cell lysates were centrifuged at $13000 \times \mathrm{g}$ 
for $20 \mathrm{~min}$ at $4{ }^{\circ} \mathrm{C}$. The supernatants were collected for protein electrophoresis. Proteins were detected using appropriate antibodies.

RNA interference. To generate stable RIP1-knockdown in HepG2/DDP-RIP3 cells, shRNA targeting RIP1 sequence (CCACTAGTCTGACGGATAA) was cloned into pshRNA vector (pshRNA-RIP1). Lentivirus was produced by co-transfecting 293T cells with pshRNA, pMD2.G, psPAX2. HepG2/ DDP-RIP3 cells were infected with the lentivirus supernatant and selected by puromycin to generate stable cell line.

In vivo xenograft assay. Briefly, $5 \times 10^{5}$ tumor cells were suspended in $100 \mu \mathrm{l}$ PBS and injected subcutaneously into nude mice $(\mathrm{n}=5)$. Tumor size was measured every 3 days with digital calipers. Tumor volume was calculated using the formula: tumor volume $\left(\mathrm{mm}^{3}\right)=0.5 \times$ (long diameter) $\times(\text { short diameter })^{2}$. PBS or cisplatin $(10 \mathrm{mg} / \mathrm{kg})$ was injected i.p. into mice every two days. All mice were maintained in a specific pathogen-free facility. All experimental manipulations were undertaken in accordance with the Institutional Guidelines for the Care and Use of Laboratory Animals, Department of Hepatobiliary Surgery, The Affiliated Hospital of Xuzhou Medical University, People's Republic of China.

\section{Results}

HepG2/DDP cells are tolerant to cisplatin treatment. To determine the sensitivity to chemotherapy reagent, HepG2 and HepG2/DDP cells were treated with different concentrations of cisplatin $(0,2,5,10$ or $20 \mu \mathrm{M})$. After 48 hours, cell viability was determined by measuring the total amount of ATP using CellTiter-Glo Luminescent Cell Viability Assay. As Figure 1A showed, lower concentration of cisplatin induced much more cell death in HepG2 cells comparing with HepG2/DDP cells, suggesting that the latter cells were more tolerant to increasing concentrations of cisplatin treatment. In time-course experiments, both HepG2 and HepG2/ DDP cells were incubated with $10 \mu \mathrm{M}$ cisplatin for $0,24,48$ and 72 hours. Starting from 24 hours post-treatment, HepG2 cells showed more significant reduction in cell viability than HepG2/DDP cells over time (Figure1B).

Previous studies have showed that upregulation of antiapoptotic genes and downregulation of apoptotic genes were responsible for the cisplatin resistance in cancer cells. Thus, we detected the expression of these apoptosis-related genes at mRNA level by real-time PCR. Our results showed that

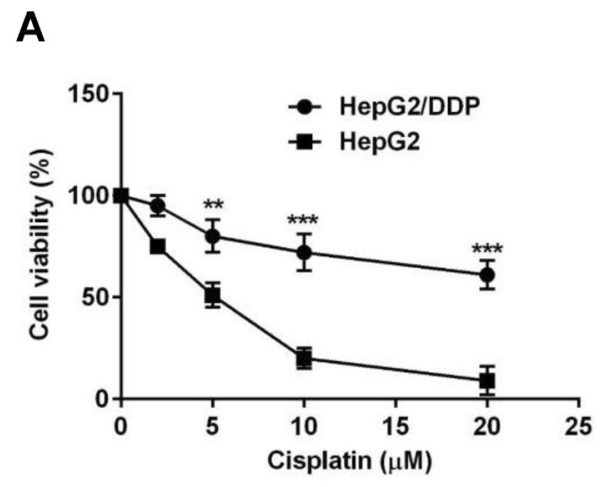

B
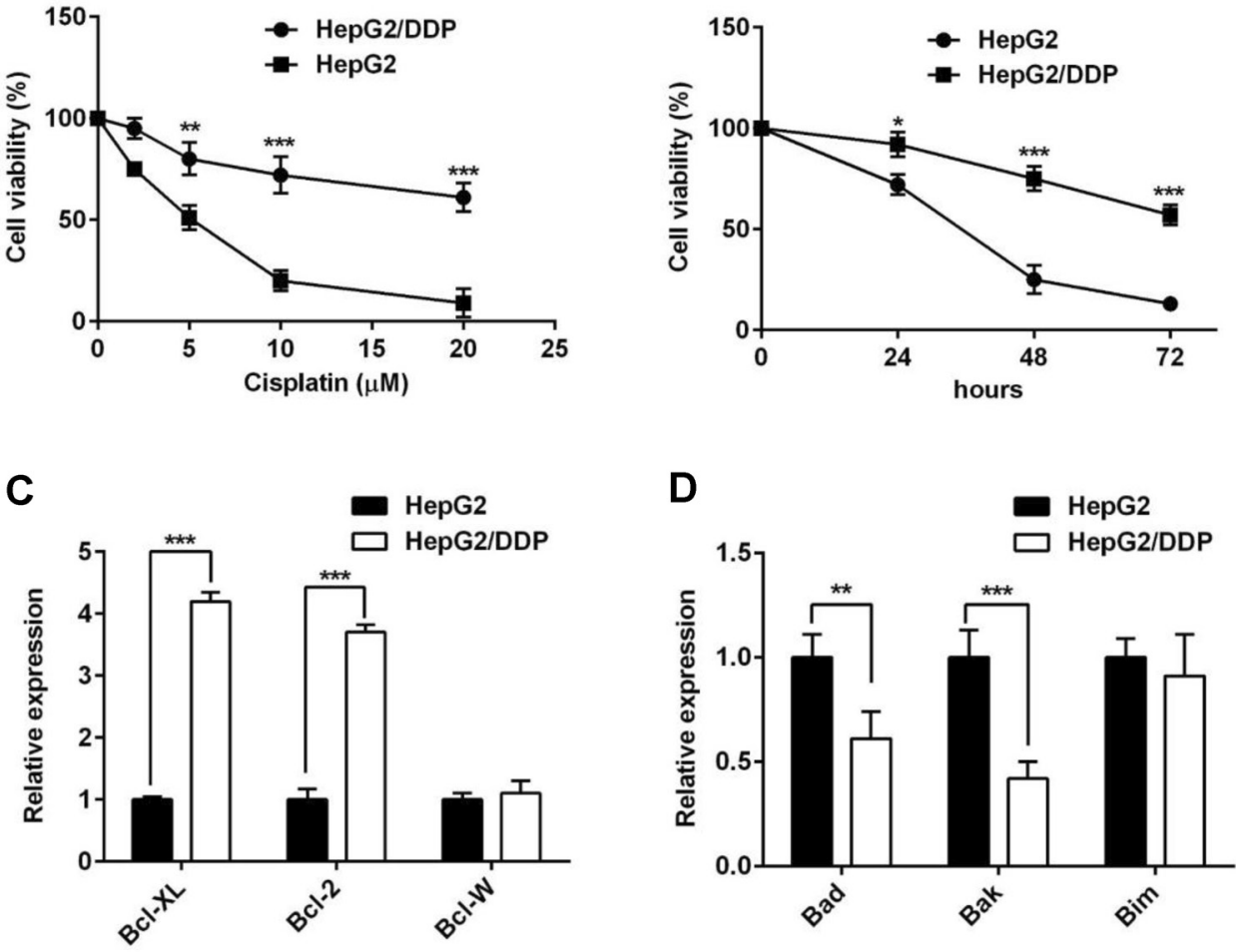

Figure 1. HepG2/DDP cells are not sensitive to cisplatin treatment. A) HepG2 and HepG2/DDP cells were treated with different concentrations of cisplatin $(0,2,5,10,20 \mu \mathrm{M})$ for 48 hours and cell viability was measured using CellTiter-Glo Luminescent Cell Viability Assay. B) HepG2 and HepG2/ DDP cells were incubated with or without $10 \mu \mathrm{M}$ cisplatin for indicated time $(0,24,48,72$ hours $)$ then the cell viability was measured using CellTiterGlo Luminescent Cell Viability Assay. Cell viability (100\%) = cisplatin-treated group/no cisplatin-treated group ${ }^{\star} 100 \%$. C, D) Real-time PCR detection of pro-Apoptotic genes (Bad, Bak and Bim) and anti-apoptotic genes (Bcl-2, Bcl-XL and Bcl-W). Representative results of at least three experiments are shown. ${ }^{*} \mathrm{p}<0.05,{ }^{* *} \mathrm{p}<0.01$ and ${ }^{* * *} \mathrm{p}<0.001$. 
expression of $\mathrm{Bcl}-\mathrm{XL}$ and $\mathrm{Bcl}-2$, which prevented apoptosis, were obviously higher in HepG2/DDP than in HepG2 cells. In addition, pro-apoptotic genes Bak and Bad were significantly decreased in HepG2/DDP cells comparing with HepG 2 cells. Other genes expressions were similar between both cell lines (Figures 1C and 1D). This result indicated that HepG2/DDP cells had evolved resistance to cisplatin by altering their apoptosis signaling pathway. Maybe some, but not all, apoptotic genes were changed during this evolution. This may be the intrinsic character of HepG2/ DDP cells. Taken together, these results demonstrated that HepG2/DDP cells were resistant to cisplatin treatment and this phenomenon was related with abnormal expression of apoptotic genes.

Lack of necroptosis in HepG2/DDP cells. Previous papers reported that upregulation of anti-apoptotic pathway in many cancer cells lead to tumor chemoresistance and suggested that activation of necroptosis could be a good therapeutic strategy choice for these cancer cells [15]. To examine whether HepG2/DDP cells responded to critical necroptosis, they were incubated with a combination of TNF- $\alpha$ plus Smac mimetic and the caspase inhibitor $\mathrm{z}$-VAD $(\mathrm{T} / \mathrm{S} / \mathrm{Z})$ to trigger necroptosis. As shown in Figure 2A, HepG2/DDP cells were resistant to $\mathrm{T} / \mathrm{S} / \mathrm{Z}$ induced necroptosis while HT-29 (a human colon cancer cell line which was widely used as the cellular model of $\mathrm{T} / \mathrm{S} / \mathrm{Z}$ induced necroptosis) underwent cell death significantly. Thus, we measured the expression levels of critical modulators (RIP1, RIP3 and MLKL) of TNF- $\alpha$ induced necroptosis in HepG2/DDP and HT-29 cell lines. RT-PCR analysis showed that the RIP1 and MLKL were ubiquitously expressed in the two cell lines, but RIP3 was only detected in the HT-29 cells (Figure 2B). Western blot analysis also confirmed RIP3 protein was deficient in HepG2/DDP cells, but present in HT-29 cells (Figure $2 \mathrm{C})$. These results may suggest that deficiency of RIP3 in HepG2/DDP cells was attributed to their resistance to $\mathrm{T} / \mathrm{S} / \mathrm{Z}$ induced necroptosis. To prove our hypothesis, we generated stable RIP3-overexpressing HepG2/DDP cell line (HepG2/DDP-RIP3) and vector transduced cells as the control (HepG2/DDP-vector). RIP3 overexpression was confirmed by western blot analysis (Figure 2D). Critically, ectopic expression of RIP3 in HepG2/DDP cells sensitized them to T/S/Z induced necroptosis (Figure $2 \mathrm{E}$ ).

Cisplatin induces necroptosis in HepG2/DDP cells by RIP3 overexpression. Previous studies revealed that some cancer cells underwent non-apoptotic cell death, such as necroptosis, in response to cisplatin $[19,20]$. In our work, we first asked what kind of cell death was induced by cisplatin in HepG2 and HepG2/DDP-RIP3 cells. As shown in Figure $3 \mathrm{~A}, \mathrm{HepG} 2$ cells were sensitive to low concentration of cisplatin treatment and the cell viability was obviously recovered when a general caspase inhibitor (zVAD) was used, indicating that cisplatin predominantly prompted caspase-dependent apoptosis in these cells. This was consistent with the findings of previous studies. Interestingly, low concentration of cisplatin also induced obvious cell death in HepG2/DDP-RIP3 cells, but not the HepG2/ DDP cells (Figure 3B). To further examine whether inhibiting caspase activity blocked cisplatin-induced cell death, the pan caspase zVAD was used. As shown (Figure 3B), zVAD treatment did not block but to some extent promoted cisplatin induced cell death in HepG2/DDP-RIP3 cells. These observations reminded us that cisplatin seemed to promote another type of cell death, necroptosis, when RIP3 was restored and the apoptosis pathway was inhibited in HepG2/DDP cells.
A

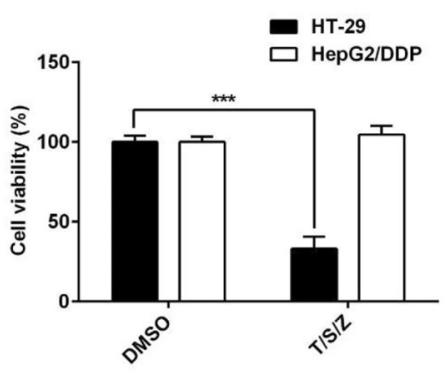

B
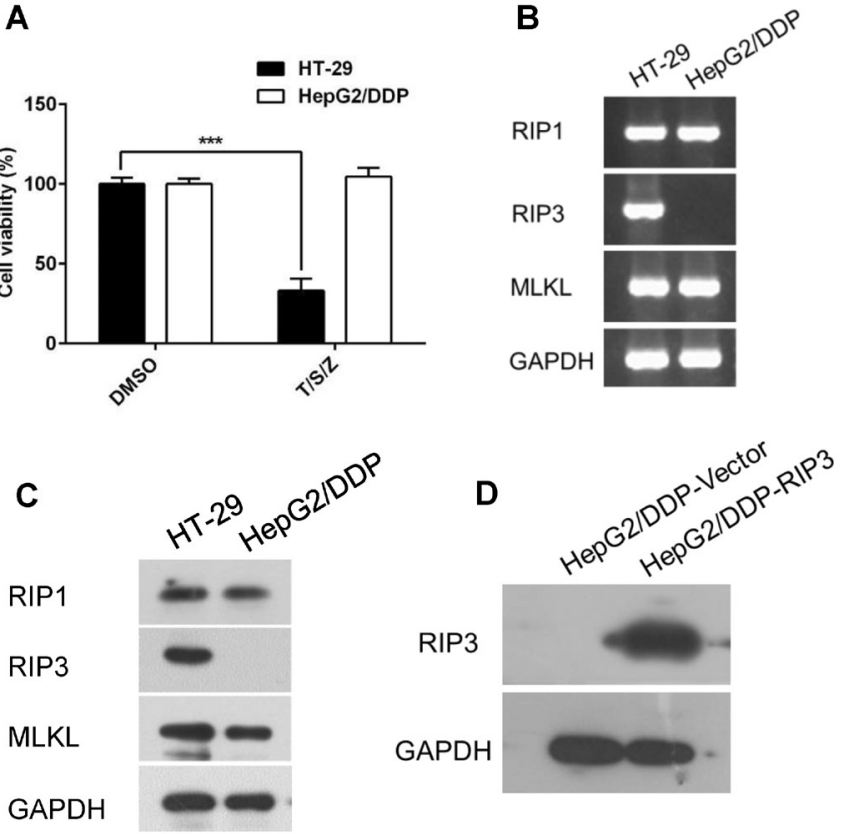

E

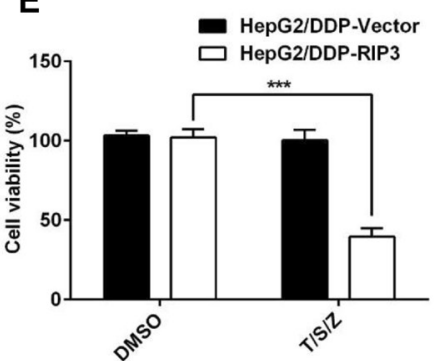

Figure 2. Absent of TNF- $\alpha$ mediated necroptosis in HepG2/DDP cells. A) HT-29 and HepG2/DDP cells were treated with or without T/S/Z for 24 hours then the cell viability was measured using CellTiter-Glo Luminescent Cell Viability Assay. RIP1, RIP3 and MLKL expression were detected at mRNA level by RT-PCR (B) and at protein level by western blot (C) in HT-29 cells and HepG2/DDP cells. D) Overexpression of RIP3 protein in HepG2/DDP-RIP3 cells was measured by western blot. E) HepG2/DDPVector and HepG2/DDP-RIP3 cells were treated with or without T/S/Z for 24 hours then the cell viability was measured using CellTiter-Glo Luminescent Cell Viability Assay. One representative of at least three experiments with similar results was shown. ${ }^{\star} \mathrm{p}<0.05,{ }^{\star *} \mathrm{p}<0.01$ and ${ }^{\star * *} \mathrm{p}<0.001$ compared with the control. 
Next we used several approaches to further prove our hypothesis. First, lactate dehydrogenase (LDH) release was measured, which was a characteristic feature of necroptosis. As shown in Figure 3C, the release of $\mathrm{LDH}$ was increased significantly after cisplatin treatment in HepG2/DDP-RIP3 cells, but not in the HepG2/DDP cells. In addition, another necroptosis marker, HMGB1 was also produced by cisplatin stimulation in RIP3 overexpression HepG2/DDP cells (Figure 3D). Collectively, these data indicated that cisplatin predominately induced non-apoptotic necroptosis in RIP3 overexpression HepG2/DDP cells.

The molecular mechanisms of cisplatin-induced necroptosis in HepG2/DDP-RIP3 cells. Previous studies have identified RIP3 as a critical modulator in T/S/Z-induced necroptosis [10]. Based on our above data, we also confirmed that RIP3 was essential in cisplatin-induced necroptosis in HepG/DDP cells. Besides RIP3, RIP1 and MLKL also played important roles during $\mathrm{T} / \mathrm{S} / \mathrm{Z}$-induced necroptosis in many cells. So we asked whether RIP1 and MLKL were also involved in cisplatin-induced necroptosis in HepG2/ DDP-RIP3 cells. A specific inhibitor of RIP1 kinase (Nec-1) was used in our experiment. As shown in Figure 4A, there was a positive correlation between concentrations of Nec-1 and HepG2/DDP-RIP3 cell viability after treatment with cisplatin. Furthermore, MLKL inhibitor (NSA) also had the same effect on cisplatin-induced cell death (Figure 4B). Furthermore, specific shRNA targeting RIP1 was used and the efficiency of RIP1 knockdown at protein level was confirmed by western blot analysis (Figure 4C). RIP1 knockdown markedly rescued cisplatin-induced cell death (Figure 4D). Taken together, these results indicated that cisplatin triggered HepG2/DDP-RIP3 cells death through RIP1/RIP3/MLKL signaling pathway.

Next we explored how cisplatin treatment activated the classic necroptosis signaling pathway. Previous studies showed that autocrine production of TNF- $\alpha$ was required for the induction of necroptosis in L929 cells [23]. We measured TNF- $\alpha$ mRNA level using real-time PCR. Our results showed

\section{A}

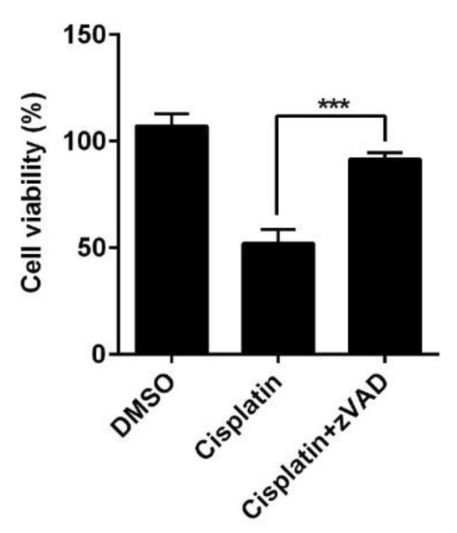

C

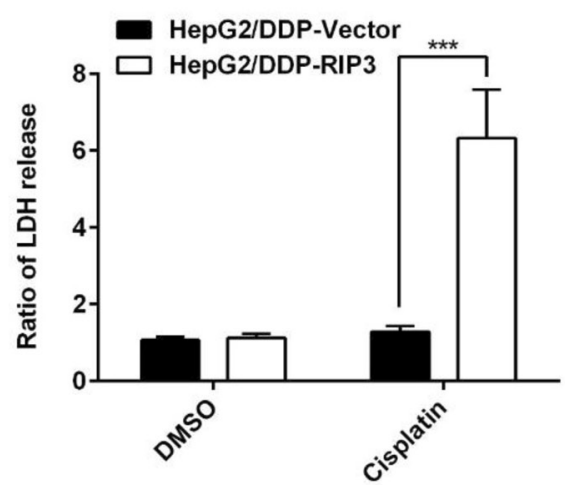

B

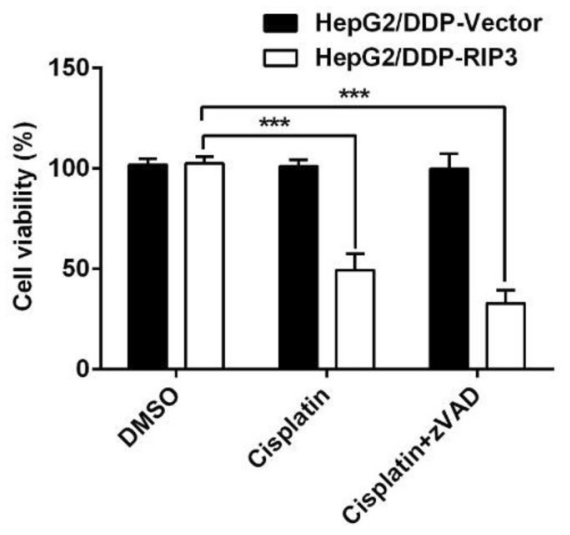

D

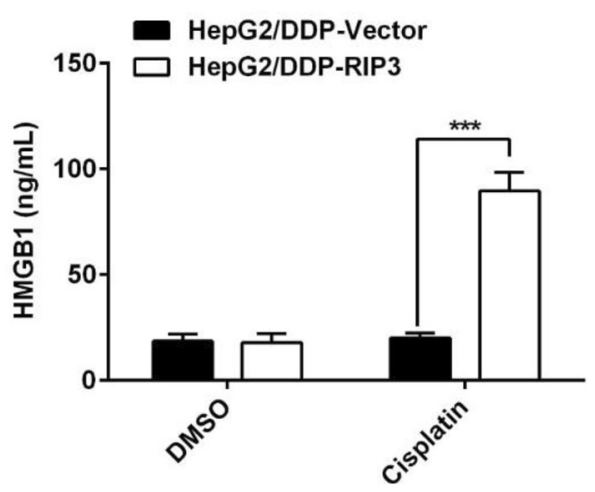

Figure 3. RIP3 overexpression promotes cisplatin-induced cell death in HepG2/DDP cells. A) HepG2 cells were treated with or without $\mathrm{zVAD}$ (20 $\mu \mathrm{M})$ for 1 hour followed by stimulation with $10 \mu \mathrm{M}$ cisplatin for 48 hours. The cell viability was measured using CellTiter-Glo Luminescent Cell Viability Assay. B) HepG2/DDP-Vector and HepG2/DDP-RIP3 cells were treated with or without zVAD $(20 \mu \mathrm{M})$ for 1 hour followed by stimulation with $10 \mu \mathrm{M}$ cisplatin for 48 hours. The cell viability was measured using CellTiter-Glo Luminescent Cell Viability Assay. LDH release (C) and HMGB1 (D) in the culture supernatants were measured after cisplatin treatment for 48 hours in HepG2/DDP-Vector and HepG2/DDP-RIP3 cells. One representative of at least three experiments with similar results was shown. ${ }^{*} \mathrm{p}<0.05,{ }^{* *} \mathrm{p}<0.01$ and ${ }^{* * *} \mathrm{p}<0.001$ compared with the control. 
transcription of TNF- $\alpha$ was profoundly enhanced in HepG2/ DDP-RIP3 cells upon cisplatin treatment (Figure 4E). These results were consistent with previous studies that production of TNF- $\alpha$ was involved in cisplatin-induced necroptosis in esophageal cancer cells [24].
Expression of RIP3 in HepG2/DDP cells enhanced the therapeutic effect of cisplatin. To explore whether the overexpression of RIP3 in HepG2/DDP cells affects tumor growth, we first analyzed cellular growth rates between vector and RIP3 overexpression HepG2/DDP cells in vitro.
A
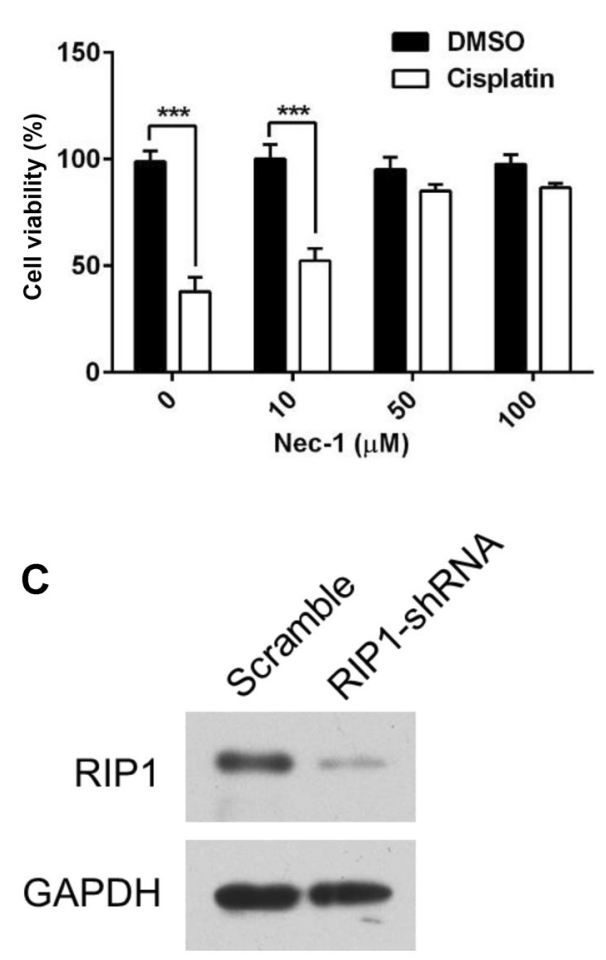

E

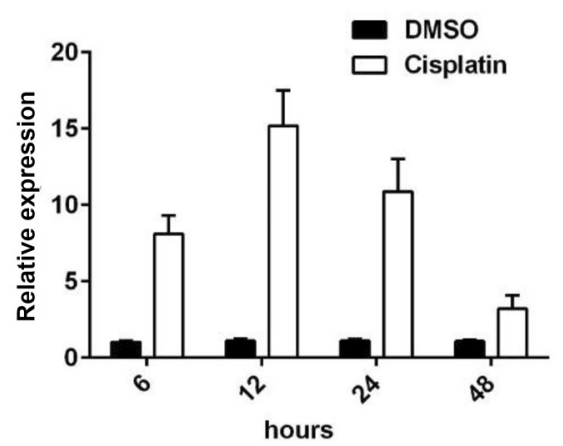

B

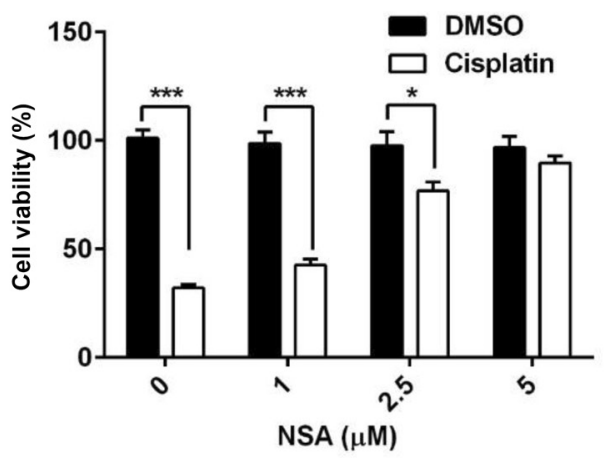

D

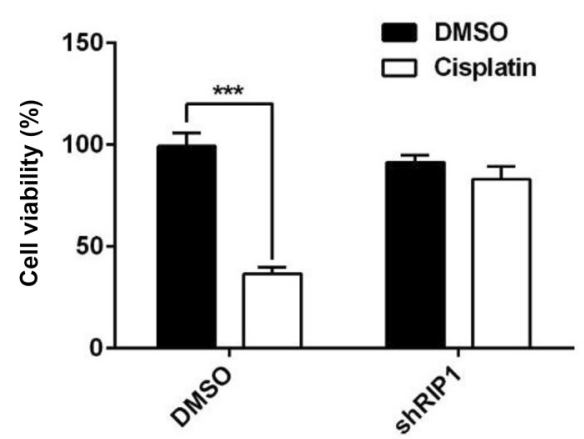

Figure 4. The molecular mechanisms of cisplatin-induced necroptosis in HepG2/DDP-RIP3 cells. HepG2/DDP-RIP3 cells were treated with different concentrations of Nec-1 (A) or NSA (B) for 1 hour followed by stimulation with $10 \mu \mathrm{M}$ cisplatin for 48 hours. The cell viability was measured using CellTiter-Glo Luminescent Cell Viability Assay. C) RIP1 expression detected by western blot in HepG2/DDP-RIP3 cells stably transfected with shRNA against RIP1. D) HepG2/DDP-RIP3 expressing RIP1 shRNA cells were stimulated with $10 \mu \mathrm{M}$ cisplatin for 48 hours. The cell viability was measured using CellTiter-Glo Luminescent Cell Viability Assay. E) HepG2/DDP-RIP3 cells were treated with $10 \mu \mathrm{M}$ cisplatin for indicated time and TNF- $\alpha$ mRNA was measured by real-time PCR. One representative of at least three experiments with similar results was shown. ${ }^{\star} \mathrm{p}<0.05,{ }^{* *} \mathrm{p}<0.01$ and ${ }^{* * *} \mathrm{p}<0.001$ compared with the control. 
The growth curve showed a 3-day doubling time of HepG2/ DDP cells and overexpression of RIP3 had no obvious effect on HepG2/DDP cells growth (Figure 5A). Moreover, we found that HepG2/DDP cells with overexpression of RIP3 showed similar tumor growth and volume in nude mice when compared with vector transduced cells (Figure 5B).

To evaluate the anti-tumor effect of cisplatin in vivo, mice were given cisplatin, which was dissolved and injected i.p. every other day at a dose of $10 \mathrm{mg} / \mathrm{kg}$ body weight when the average tumor volume reached $300 \mathrm{~mm}^{3}$ in xenograft tumor model. As shown in Figure 5C, the xenograft tumor of HepG2/DDP-RIP3 was much more sensitive to cisplatin treatment and exhibited suppressed tumor growth and size comparing with the vector transduced HepG2/DDP cells. Above data indicated that ectopic expression of RIP3 sensitized HepG2/DDP cancer cells to cisplatin treatment in vivo.

\section{Discussion}

Cisplatin is one of the most important chemotherapeutic agents for the treatment of solid tumors. Cisplatin cytotoxicity is mediated by DNA damage and the subsequent induction of type I cell death, apoptosis [25]. Induction of apoptosis in tumor cells by many chemotherapy drugs (e.g., cisplatin, 5 -fluorouracil) is the major strategy for cancer treatment [26]. But cancer cells always evolve to avoid apoptosis, resulting in drug resistance and the ineffectiveness of anti-cancer therapies. In this study, two cell lines were used: HepG2 and HepG2/DDP. Consistent with previous report [27], HepG2 cells, but not HepG2/DDP cells, were sensitive to cisplatin treatment as low concentration of cisplatin could induce obvious cell death. Cisplatin resistance in HepG2/DDP cells may result from upregulation of anti-apoptotic genes (Bcl-XL and $\mathrm{Bcl}-2)$ and decreased expression of pro-apoptotic genes (Bad and Bax) (Figure 1).

Recently, necroptosis had been proposed to be a new strategy for cancer therapy [15]. Necroptosis had been described for many years as an accidental and uncontrolled form of cell death due to chemical agent, oxidative stress and mechanical damage [28]. Now necroptosis is considered as an important form of programmed cell death and the molecular signaling pathway during cell necroptosis is increasingly realized. Necroptosis can be mediated by TNF receptor superfamily, T-cell receptors, Toll-like receptors, cellular metabolic stresses or anticancer compounds [29]. Necroptosis and apoptosis use distinct molecular signaling pathways and
A

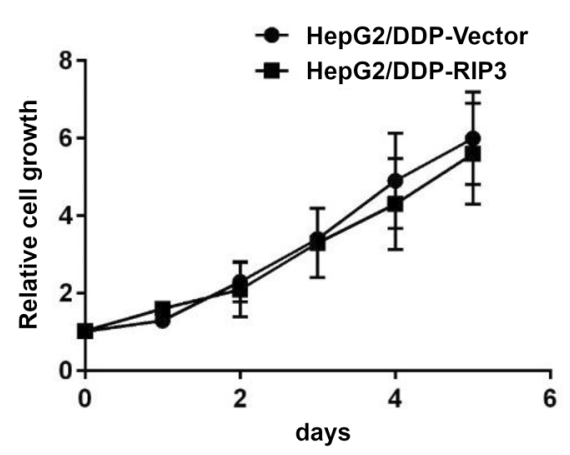

B

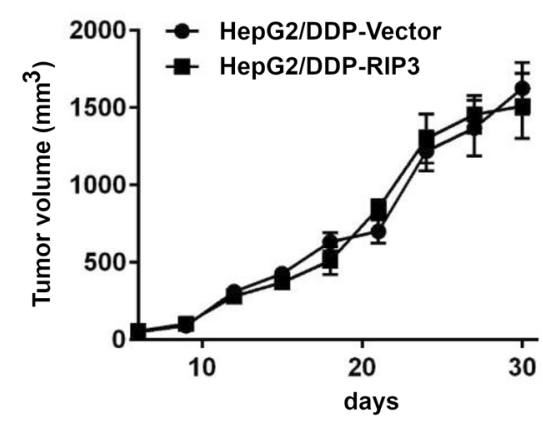

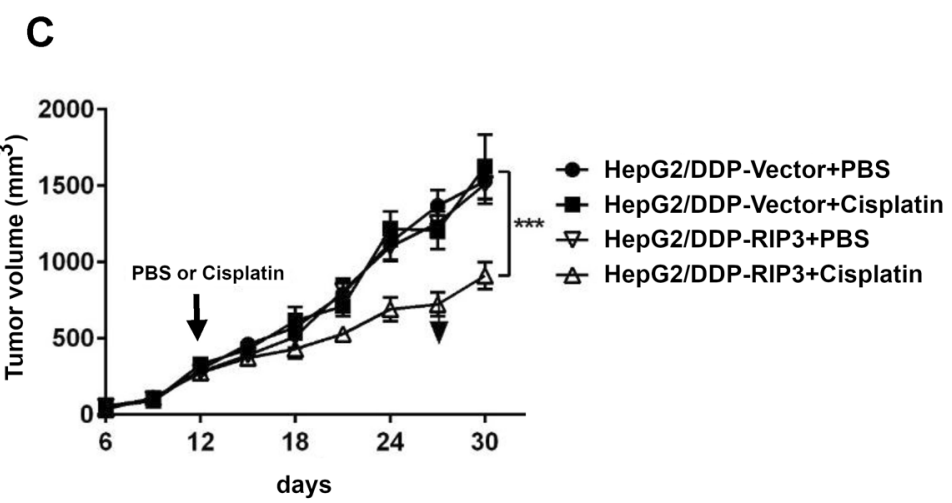

Figure 5. HepG2/DDP-RIP3 cells were sensitive to cisplatin therapy in vivo. A) Growth curve of HepG2/DDP-Vector and HepG2/DDP-RIP3 cells as measured by CellTiter-Glo Luminescent Cell Viability Assay. B) Xenograft tumor growth curves in nude mice from HepG2/DDP-Vector and HepG2/ DDP-RIP3 cells. $n=6$ per group. C) HepG2/DDP-Vector and HepG2/DDP-RIP3 cells were subcutaneously inoculated in nude mice. When the tumor volume reached $300 \mathrm{~mm}^{3}$, mice were injected i.p. with PBS or cisplatin $(10 \mathrm{mg} / \mathrm{kg})$ every other day. $\mathrm{n}=5$ per group. ${ }^{* * *} \mathrm{p}<0.001$ compared with PBS injected group. 
necroptosis is usually activated when the apoptosis pathway is inhibited or absent in cancer cells. So induction of necroptosis could be an alternative way for apoptosis-resistant cancer therapy. This hypothesis was demonstrated in many cancer cells like HT-29, Caco-2 and CT26 [30, 31]. To explore whether HepG2/DDP could also undergo necroptosis, they were treated with classic necroptosis inducer: TNF- $\alpha$ plus Smac mimic and zVAD. Unfortunately, no obvious cell death was observed under this condition. The deficient necroptotic machinery may attribute to the absence of RIP3 expression in HepG2/DDP cells (Figure 2). So we overexpressed RIP3 protein in HepG2/DDP cells and the HepG2/DDP-RIP3 cells became sensitive to TNF- $\alpha$ plus Smac mimic and zVAD treatment. Moreover, we found that the viability of HepG2/ DDP-RIP3 cells, but not HepG2/DDP cells, was susceptible to cisplatin treatment and caspase-independent cell death was observed (Figure 3). The release of LDH and HMGB1, the classic biomarkers of necroptosis, were also detected in the supernatant of cisplatin treated HepG2/DDP-RIP3 cells (Figure 3). Based on above observation, we may conclude that cisplatin may trigger necroptosis in RIP3-overexpressed HepG2/DDP cells. To further confirm that RIP3 expression made cells sensitive to cisplatin treatment, we used L929 cells, which expressed endogenous RIP1, RIP3 and MLKL. As shown in Figure S1, cisplatin treatment induced obvious cell death in L929 cells. In addition, Nec-1 (RIP1 inhibitor) or GSK'872 (RIP3 inhibitor) can partially rescue cisplatin induced cell death. So we reasoned that cisplatin can induce two types of cell death (apoptosis and necroptosis) in L929 cells, which had endogenous RIP3 expression. Nec-1 or GSK'872 only inhibited cisplatin-induced necroptosis, but not apoptosis.

Recent research had uncovered the core components during TNF- $\alpha$-induced programmed necroptosis including RIP1, RIP3 and MLKL. RIP1 and RIP3 formed a complex (necrosome) to activate downstream executing molecule MLKL leading to necrotic cell death [32]. RIP1 kinase activity was required for RIP3 activation, and subsequently, RIP3 recruited and phosphorylated MLKL at Ser345, a key event in the activation and trimerization of MLKL. MLKL trimer then translocated to the plasma membrane and caused plasma membrane permeabilization [1]. Necroptosis can be inhibited by chemical compounds, such as RIP1 kinase inhibitor (Nec-1), RIP3 kinase inhibitor (GSK'872) and MLKL inhibitor (NSA) [16]. However, it was unclear whether cisplatininduced cell death was also associated with RIP1-RIP3-MLKL signaling pathway. In this study, our data demonstrated that RIP3 was indispensable for cisplatin-induced necroptosis in HepG2/DDP cells, which was primarily executed through RIP1-RIP3-MLKL signaling pathway in HepG2/DDP-RIP3 cells. In addition, our results suggested that RIP1 was necessary for cisplatin-induced necroptosis because knockdown of RIP1 by shRNA or pharmacological inhibition of RIP1 by Nec-1 prominently attenuated cisplatin-induced necroptosis in the HepG2/DDP-RIP3 cells. In addition, MLKL inhibitor
NSA almost totally prohibited cisplatin-induced necroptosis in HepG2/DDP-RIP3 cells (Figure 4). Our data provided new insights into the mechanism of cisplatin-induced cell death in HepG2/DDP cells. Further, previous reports showed that autocrine release of TNF- $\alpha$ was identified in cisplatininduced cell necroptosis in acute renal failure and in esophageal cancer cells. We also observed enhanced TNF- $\alpha$ mRNA synthesis, suggesting that cisplatin-induced necroptosis in HepG2/DDP-RIP3 cells was initiated by the autocrine production of TNF- $\alpha$.

In this study, we found that overexpression of RIP3 in the HepG2/DDP cells did not affect tumor cell growth in vitro. In the mouse xenograft model, cisplatin therapy was more effective in reducing tumor volume in HepG2/DDP-RIP3 cells comparing with the HepG2/DDP cells. On the one hand, cisplatin treatment may induce HepG2/DDP-RIP3 cells necroptosis in vivo then inhibit tumor growth. On the other hand, necroptosis was considered as a type of immunogenic cell death, which elicited a specific immune response to promote killing cancer cells [33]. This study also suggested that the high expression level of RIP3 in tumor was associated with better response rates to cisplatin clinical therapy in patients. However, RIP3 expression was usually repressed in liver tumor cells and $85 \%$ of breast cancer patients due to genomic methylation. So RIP3-dependent activation of necroptosis was also largely inhibited during cancer chemotherapy. Previous study showed that hypomethylating agents could restore RIP3 expression in some tumor cells [34], promoting their sensitivity to chemotherapeutics-induced cell necroptosis. Therefore, patients may benefit from the combined use of chemotherapeutics and hypomethylating agents in clinical trials.

In summary, we confirmed the upregulation of antiapoptotic molecules by qPCR that blocked the apoptotic cell death in HepG2/DDP cells in response to cisplatin. HepG2/DDP cells also failed to undergo necroptosis due to deficiency of RIP3 expression, suggesting that RIP3 was essential for cisplatin-induced necroptosis in HepG2/ DDP cells. In addition to RIP3, our data indicated that RIP1, MLKL and synthesis of TNF- $\alpha$ contributed to cisplatin-initiated necroptosis in HepG2/DDP cells. Finally, we demonstrated that HepG2/DDP cells were more sensitive to cisplatin treatment in xenograft tumor model. Our results indicated that triggering of necroptosis was an alternative strategy for killing apoptosis-resistant tumor cells. A better understanding of the necroptosis machinery induced by cisplatin was extremely important for improving cancer chemotherapy. However, whether hypomethylating agents can upregulate of RIP3 expression in patients was unclear. The therapeutic effect of the combination of hypomethylating agents and chemotherapeutics need to be studied thoroughly in future. In addition, RIP3 expression was normal in human tissue. We must monitor the side effects of chemotherapeutics, which may also induced RIP3-dependent necroptosis in normal tissues. 
Supplementary information is available in the online version of the paper.

\section{References}

[1] NIKOLETOPOULOU V, MARKAKI M, PALIKARAS K, TAVERNARAKIS N. Crosstalk between apoptosis, necrosis and autophagy. Biochim Biophys Acta 2013; 1833: 34483459. https://doi.org/10.1016/j.bbamcr.2013.06.001

[2] FUCHS Y, STELLER H. Programmed cell death in animal development and disease. Cell 2011; 147: 742-758. https:// doi.org/10.1016/j.cell.2011.10.033

[3] ICHIM G, TAIT SW. A fate worse than death: apoptosis as an oncogenic process. Nat Rev Cancer 2016; 16: 539-548. https://doi.org/10.1038/nrc.2016.58

[4] NAGATA S. Apoptotic DNA fragmentation. Exp Cell Res 2000; 256: 12-18. https://doi.org/10.1006/excr.2000.4834

[5] KARCH J, MOLKENTIN JD. Regulated necrotic cell death: the passive aggressive side of Bax and Bak. Circ Res 2015; 116: 1800-1809. https://doi.org/10.1161/CIRCRESAHA.116.305421

[6] MOQUIN D, CHAN FK. The molecular regulation of programmed necrotic cell injury. Trends Biochem Sci 2010; 35: 434-441. https://doi.org/10.1016/j.tibs.2010.03.001

[7] VANLANGENAKKER N, VANDEN BERGHE T, KRYSKO DV, FESTJENS N, VANDENABEELE P. Molecular mechanisms and pathophysiology of necrotic cell death. Curr Mol Med 2008; 8: 207-220. https://doi. org/10.2174/156652408784221306

[8] FINK SL, COOKSON BT. Apoptosis, pyroptosis, and necrosis: mechanistic description of dead and dying eukaryotic cells. Infect Immun 2005; 73: 1907-1916. https://doi. org/10.1128/IAI.73.4.1907-1916.2005

[9] MCCALL K. Genetic control of necrosis - another type of programmed cell death. Curr Opin Cell Biol 2010; 22: 882888. https://doi.org/10.1016/j.ceb.2010.09.002

[10] HE S, WANG L, MIAO L, WANG T, DU F et al. Receptor interacting protein kinase-3 determines cellular necrotic response to TNF-alpha. Cell 2009; 137: 1100-1111. https://doi. org/10.1016/j.cell.2009.05.021

[11] ZHANG DW, SHAO J, LIN J, ZHANG N, LU BJ et al. RIP3, an energy metabolism regulator that switches TNF-induced cell death from apoptosis to necrosis. Science 2009; 325: 332-336. https://doi.org/10.1126/science.1172308

[12] Seifert L, Miller G. Molecular Pathways: The Necrosome-A Target for Cancer Therapy. Clin Cancer Res 2017; 23: 11321136. https://doi.org/10.1158/1078-0432.CCR-16-0968

[13] SUN L, WANG H, WANG Z, HE S, CHEN S et al. Mixed lineage kinase domain-like protein mediates necrosis signaling downstream of RIP3 kinase. Cell 2012; 148: 213-227. https://doi.org/10.1016/j.cell.2011.11.031

[14] CHEUNG-ONG K, GIAEVER G, NISLOW C. DNAdamaging agents in cancer chemotherapy: serendipity and chemical biology. Chem Biol 2013; 20: 648-659. https://doi. org/10.1016/j.chembiol.2013.04.007

[15] SU Z, YANG Z, XIE L, DEWITT JP, CHEN Y. Cancer therapy in the necroptosis era. Cell Death Differ 2016; 23: 748-756. https://doi.org/10.1038/cdd.2016.8
[16] MENG MB, WANG HH, CUI YL, WU ZQ, SHI YY et al. Necroptosis in tumorigenesis, activation of anti-tumor immunity, and cancer therapy. Oncotarget 2016; 7: 5739157413. https://doi.org/10.18632/oncotarget.10548

[17] LIANG W, CAI A, CHEN G, XI H, WU X et al. Shikonin induces mitochondria-mediated apoptosis and enhances chemotherapeutic sensitivity of gastric cancer through reactive oxygen species. Sci Rep 2016; 6: 38267. https://doi. org/10.1038/srep38267

[18] HUANG C, LUO Y, ZHAO J, YANG F, ZHAO H et al. Shikonin kills glioma cells through necroptosis mediated by RIP-1. PLoS One 2013; 8: e66326. https://doi.org/10.1371/journal. pone. 0066326

[19] ZHANG LJ, HAO YZ, HU CS, YE Y, XIE QP et al. Inhibition of apoptosis facilitates necrosis induced by cisplatin in gastric cancer cells. Anticancer Drugs 2008; 19: 159-166. https://doi.org/10.1097/CAD.0b013e3282f30d05

[20] XU Y, LIN Z, ZHAO N, ZHOU L, LIU F et al. Receptor interactive protein kinase 3 promotes Cisplatin-triggered necrosis in apoptosis-resistant esophageal squamous cell carcinoma cells. PLoS One 2014; 9: e100127. https://doi.org/10.1371/ journal.pone.0100127

[21] Fernald K, Kurokawa M. Evading apoptosis in cancer. Trends Cell Biol 2013; 23: 620-633. https://doi.org/10.1016/j. tcb.2013.07.006

[22] FANG D, GUO Y, ZHU Z, CHEN W. Silence of p15 expression by RNAi enhances cisplatin resistance in hepatocellular carcinoma cells. Bosn J Basic Med Sci 2012; 12: 4-9. https:// doi.org/10.17305/bjbms.2012.2523

[23] WU YT, TAN HL, HUANG Q, SUN XJ, ZHU X et al. zVADinduced necroptosis in L929 cells depends on autocrine production of TNFalpha mediated by the PKC-MAPKs-AP-1 pathway. Cell Death Differ 2011; 18: 26-37. https://doi. org/10.1038/cdd.2010.72

[24] XU Y, MA HB2, FANG YL3, ZHANG ZR2, SHAO J et al. Cisplatin-induced necroptosis in TNFalpha dependent and independent pathways. Cell Signal 2017; 31: 112-123. https://doi.org/10.1016/j.cellsig.2017.01.00

[25] GUMULEC J, BALVAN J, SZTALMACHOVA M, RAUDENSKA M, DVORAKOVA V et al. Cisplatin-resistant prostate cancer model: Differences in antioxidant system, apoptosis and cell cycle. Int J Oncol 2014; 44: 923-933. https://doi.org/10.3892/ijo.2013.2223

[26] MHAIDAT NM, BOUKLIHACENE M, THORNE RF. 5-Fluorouracil-induced apoptosis in colorectal cancer cells is caspase-9-dependent and mediated by activation of protein kinase C-delta. Oncol Lett 2014; 8: 699-704. https://doi. org/10.3892/ol.2014.2211

[27] LIU XY, LIU SP, JIANG J, ZHANG X, ZHANG T. Inhibition of the JNK signaling pathway increases sensitivity of hepatocellular carcinoma cells to cisplatin by down-regulating expression of P-glycoprotein. Eur Rev Med Pharmacol Sci 2016; 20: 1098-1108.

[28] KROEMER G, EL-DEIRY WS, GOLSTEIN P, PETER ME, VAUX D et al. Classification of cell death: recommendations of the Nomenclature Committee on Cell Death. Cell Death Differ 2005; 12 Suppl 2: 1463-1467. https://doi.org/10.1038/ sj.cdd. 4401724 
[29] SU Z, YANG Z, XU Y, CHEN Y, YU Q. Apoptosis, autophagy, necroptosis, and cancer metastasis. Mol Cancer 2015; 14 : 48. https://doi.org/10.1186/s12943-015-0321-5

[30] TAKEMURA R, TAKAKI H, OKADA S, SHIME H, AKAZAWA $T$ et al. PolyI:C-Induced, TLR3/RIP3-Dependent Necroptosis Backs Up Immune Effector-Mediated Tumor Elimination In Vivo. Cancer Immunol Res 2015; 3: 902-914. https://doi.org/10.1158/2326-6066.CIR-14-0219

[31] KAKU Y, TSUCHIYA A, SHIMIZU T, TANAKA A, NISHIZAKI T. HUHS1015 Suppresses Colonic Cancer Growth by Inducing Necrosis and Apoptosis in Association with Mitochondrial Damage. Anticancer Res 2016; 36: 3948.
[32] YANG CK, HE SD. Heat shock protein 90 regulates necroptosis by modulating multiple signaling effectors. Cell Death Dis 2016; 7: e2126. https://doi.org/10.1038/cddis.2016.25

[33] GARG AD, AGOSTINIS P. Editorial: Immunogenic Cell Death in Cancer: From Benchside Research to Bedside Reality. Front Immunol 2016; 7: 110. https://doi.org/10.3389/ fimmu.2016.00110

[34] KOO GB, MORGAN MJ, LEE DG, KIM WJ, YOON JH et al. Methylation-dependent loss of RIP3 expression in cancer represses programmed necrosis in response to chemotherapeutics. Cell Res 2015; 25: 707-725. https://doi.org/10.1038/ cr.2015.56 